\section{Free recall of trigrams following their presentation in a short-term memory task*}

\author{
LAURENCE MILLER, WHLLIAM ADAMS, JERRY DEFFENBACHER \\ and LARRY HALL \\ Western Washington State College, Bellingham, Wash. 98225
}

Ss free recalled items they had previously separately verbally recalled in a standard short-term memory task. Free recall was examined as a function of: (1) order of item presentation, (2) length of retention interval, (3) presence or absence of an instructional set to recall all items, and (4) activity during the intertrial interval. Significant effects due to order of item presentation, intertrial-interval activity, and interactions between order of presentation and intertrial-interval activity and between presentation order and instructions were found. A recency effect appeared in all instances, but a primacy effect was obtained only when Ss were forewarned of free recall. The effect of intertrial-interval activity was most evident with the last item. Rehearsal of the item produced better recall than either counting or doing nothing, but counting produced better recall than doing nothing.

Free recall of consonant trigrams following their verbal recall in a standard short-term memory paradigm was examined. This procedure differs from standard free recall because: Ss try first to recall verbally each item following a retention interval; there is an appreciable intertrial interval (ITI) between each item; and a small number (six) of items was used, considering total presentation time (about $3 \mathrm{~min}$ ). Free recall was examined as a function of several variables which affect short-term verbal recall: presentation order of the items, the items' retention intervals, Ss' activity during the I'TI, and presence or absence of an instructional set to free recall all items (c.f. Miller, Adams, Deffenbacher, \& Hall, 1970). METHOD

The Ss, materials, procedure, and results of verbal recall are described in detail elsewhere (Miller et al, 1970). First, $288 \mathrm{Ss}$ tried to recall verbally each of the six consonant trigrams ( $31 \%$ association value; Witmer, 1935 ) separately following a 3 - or 18-sec retention interval. Ss counted backward by threes during the retention interval to prevent rehearsal, and a 15-sec ITI separated each item presentation. Half of the Ss were given the intervals in the order of 3-18-3-18-3-18 and the other half in the reverse order, to equalize total exposure duration.

Half of the Ss in each retention order were instructed to write down as many of the trigrams as possible, in any order, on the paper in front of them when three dots (...) appeared on the viewing screen. The dots

\footnotetext{
* The authors thank Dr. B. L. Kintz for his kind assistance with the statistical analysis.
}

appeared $15 \mathrm{sec}$ after Ss' guess at the sixth item. The other Ss were not told or given anything until 15 sec after their guess at Item 6.

Task-related activity during these ITIs was minimized or facilitated by having Ss count backward by threes, sit silently, or repeat aloud the prior correct trigram, which was read to them by $E$, regardless of correctness of verbal recall. For Ss who rehearsed the correct item, written free recall was considered correct only if it matched the rehearsed trigram. Since Ss in each no-rehearsal condition were not given feedback about correctness of verbal recall, free recall of the correct letters, but not in proper sequence, was counted as correct if this also happened during verbal recall. This happened only a couple of times.

RESULTS AND DISCUSSION

Since each order of retention intervals (18-3 or 3-18) was alternated across Ss, the data from each two successive Ss was combined to provide a measure of written free recall for of item presentation and instructions (Panel B). each of the six items. Analysis of variance revealed significant differences $(p<.05)$ for type of activity during the ITI $[\mathrm{F}(2,138)=$ $9.01, p<.01]$, order of item presentation $[\mathrm{F}(5,690)=76.17$, $\mathrm{p}<.01]$, and for the interactions between item order and ITI activity $[F(10,690)=3.04, \quad p<.01]$ and between item order and instructions $[F(5,690)=6.99, p<.01]$. These interactions are presented in Fig. 1 , Panels $A$ and $B$, respectively. The numbers above each data point in Panel B are explained later.

A recency effect was obtained in all instances: recall of the last (sixth) item was significantly better $(p<.01)$ than recall of Items 1-5. However, a primacy effect was found only with Ss given the set for free recall (Panel B). Recall of Item 1 was significantly better $(p<.01$ or .05$)$ than recall of Items 2-4 and was not significantly different from Item 5. Recall for Ss not given the set declined steadily before leveling off at Item 2 . Item 1 recall was not significantly better than recall of Items 2-6. Thus, the serial position effect-marked recency effect, flat middle section, and primacy effect-was found only when Ss were forewarned of the written free recall. It is also apparent that the superior recall obtained with the set as compared to no set at Items 1 and 2 was correlated with inferior recall for Items 3-6. The difference at Item 6 was significant $(p<.01)$. The set thus heightened attention to, and recall of, the initial items at the expense of the middle and end items.

The effect of the instructional set is also evident by examining the number above each data point in Panel $B$. These numbers represent mean order of free recall. A recalled item received from 1 to 6 points, depending upon whether the item was written down either first ( 1 point) through last $(6$ points). Six items was the most ever

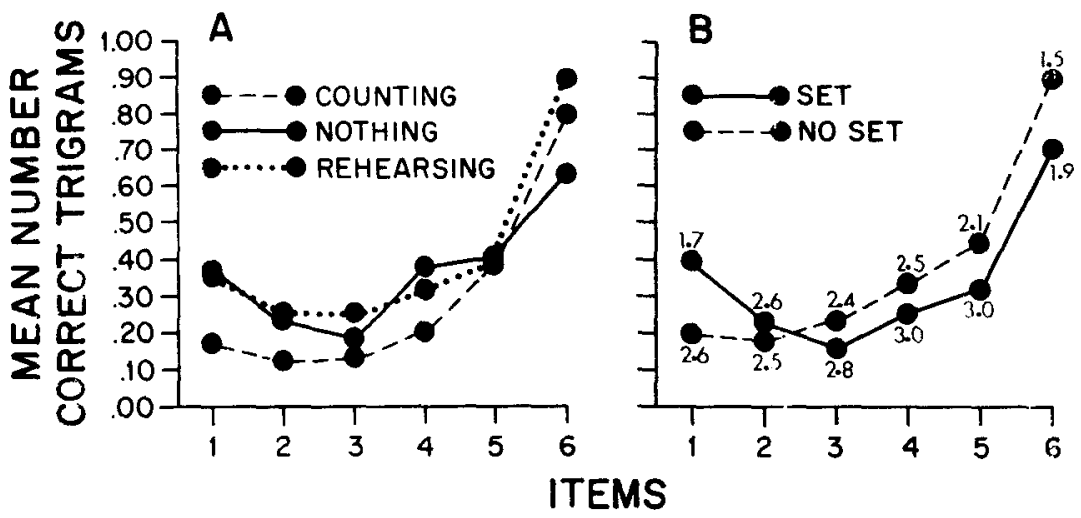

Fig. 1. Mean number of items recalled as a function of the interactions between order of item presentation and ITI activity (Panel A) and between order 
recalled. For the no-set condition, only Item 6 was recalled noticeably sooner than Items 1-5, which were all about equal. However, for the set condition, recall order for Items 1 and 6 was about equal, even though Item 6 was recalled significantly more often.

Concerning Ss' activity during the ITI, counting was correlated with poorest recall for the first four items, while the nothing and rehearsal conditions were about equal. However, none of the differences between any of the three activities at each of these four items was significant. At Item 5, recall was nearly equal. At Item 6, significantly more items were recalled with either counting or rehearsal than with nothing $(\mathrm{p}<.025$ and .01 , respectively), and significantly more $(p<.01)$ items were recalled with rehearsal than with counting. The poorer recall with counting for Items 1-4 was probably due to the counting minimizing any task-related activity, which, of course, would be available to the other two groups. The marked increase in recall at Items 5 and 6 with counting was probably due to recency of the items and lack of interference from recall of prior items. Recall was greatest for Item 6 with rehearsal, since that item was emitted by Ss until free recall began.

Thus, the short-term memory paradigm provides a useful method for not only studying item-by-item recall, but also free recall of all items presented.

\section{REFERENCES}

M I L L E R, L., A D A M S, W. DEFFENBACHER, J., \& HALL, L. The effect of instructional set and intertrial activity on retention in a short-term memory task. Psychological Record, $1970,20,373-384$.

WITMER, L. R. The association value of three-place consonant syllables. Journal of Genetic Psychology, 1935, 47, 337-360. 